\title{
Aeroacoustic Investigation of a Cavity with and without Doors by Delayed Detached Eddy Simulation
}

\author{
Yu Liu* and Mingbo Tong** \\ College of Aerospace and Engineering, Nanjing University of Aeronautics and Astronautics, Nanjing 210016, China
}

\begin{abstract}
In the present study, an effort was made to numerically investigate rectangular cavity aeroacoustics with and without doors. The simulation was performed on an open cavity with an aspect ratio of 5:1:1 at Mach 0.85 using the delayed detached eddy simulation (DDES) approach based on the Spalart-Allmaras model. Two cavity configurations, a clean cavity and a cavity with doors, were modeled. The results obtained from the clean cavity were compared with the experimental sound pressure levels (SPL) and the root mean square for the pressures applied. Furthermore, comparisons of frequencies were made using a modified semi-empirical Rossiter formula. The simulation using DDES precisely predicted the pressure fluctuation and the results matched the experiment quite well. The SPLs at the rear of the cavity were much higher than those in the front due to the instability of the shear layer impinging on the rear wall. Comparisons of DDES for the clean cavity and the doors-on cavity revealed that the SPLs inside the cavity as well as the magnitude of tones are amplified by the side doors. The main focus of this investigation was to obtain a better understanding of the open cavity acoustic resonance phenomenon and investigate the effects of cavity doors on the SPL.
\end{abstract}

Key words: DDES, Aeroacoustic, Cavity, Sound Pressure Level

\section{Introduction}

Cavity flow has been an attractive research area since Krishnamurty [1] performed acoustic investigation on twodimensional cavity configurations in 1955. The research on cavity flow is mainly about experimental, computational and analytical investigations including various types of configurations and range from subsonic to supersonic. Most research focus on the aerodynamic noise generated from the cavity, called an aeroacoustic phenomenon. The aeroacoustic resonance arises from the feedback mechanism inside the cavity. The feedback mechanism begins with the growth of shear layer instabilities from the leading edge. The high speed flow then impinges on the rear wall, generating pressure waves that propagate to the leading edge and exchange energy with the free shear layer, leading to further and periodic impact on the rear wall of the cavity. When the frequency and phase of the feedback pressure wave are consistent with the free shear layer, resonance occurs. The schematic of this feedback mechanism is shown in Fig. 1. The self-sustained cavity oscillations can induce aerodynamic noise with a level up to $170 \mathrm{~dB}$, potentially causing structural damage and failure of electronic equipment [2]. As a result, the aeroacoustic behavior of cavity flows is of key importance in the design of weapon bays and landing gear wheel wells for combat aircraft and UAV. On the other hand, in vehicles, open sunroofs and windows are cavity configurations that can generate very loud and uncomfortable noise when the vehicle moves at a certain speed.

This feedback process was first described by Rossiter [3], who developed a semi-empirical formula to predict resonant tone frequencies in various rectangular cavities from subsonic to transonic regimes in 1964. The research is commonly used in further investigations and some researchers have since modified Rossiter's equation, for example, Heller et al.s [4] modification is given in Eq. 1 .
This is an Open Access article distributed under the terms of the Creative Commons Attribution Non-Commercial License (http://creativecommons.org/licenses/by$\mathrm{nc} / 3.0 /$ ) which permits unrestricted non-commercial use, distribution, and reproduction in any medium, provided the original work is properly cited. (c) * Ph. D Student, Corresponding author: yuliunuaa@163.com ** Professor 


$$
S t_{m}=\frac{f_{m} L}{U_{\infty}}=\frac{m-\alpha}{M_{\infty} / \sqrt{1+\frac{\gamma-1}{2} M_{\infty}^{2}}+\frac{1}{K}}
$$

Where $f_{m}$ is the frequency of an acoustic tone mode, $m$ is the mode number, $\alpha$ and $\mathrm{K}$ are empirical values, $\mathrm{St}$ is the Strouhal number, $\mathrm{L}$ is the cavity length, $\mathrm{k}=0.57, \alpha=0.062^{*} \mathrm{~L} / \mathrm{D}$ and $\mathrm{D}$ is the cavity depth.

The aeroacoustic phenomenon in an open cavity is caused by the shear layer instabilities over the cavity, so simulating the shear layer using the appropriate turbulence model is very important. As the most convenient CFD method, RANS equations are the first choice for cavity acoustic behavior analyses [5-7]. However, some small scales of vortices that also have great distribution on generating acoustic waves are inaccurately captured by the RANS equation, especially in large separated flow regions. As a typical large separated flow, cavity flow can be perfectly represented by the LES method, but some investigations [8,9] revealed that the computation was too expensive when resolving the boundary and shear layers over the cavity to be a viable method for aeroacoustic analyses. In recent investigations, the DES [10-12] method proposed by Shur et al [13] has become a popular topic. Being a hybrid LES/RANS model, the DES method [14] solves the problem by adopting the RANS model in boundary layer regions and the LES model in the regions far from the wall [15]. By comparing the numerical data with experimental data, investigators found that both DES and LES performed better than RANS in resolving the vortices and velocity distributions within the cavity and the traditional RANS methods were unable to predict the broadband contribution. However, DES is preferred over LES because of its computational affordability.

Many studies were conducted with different flow conditions over various cavity configurations ranging from subsonic to supersonic. Zhang et al [16] experimentally and computationally investigated compressible flows over cavities with a wide range of L/D. Chung [17] performed experiments to study the characteristics of compressible cavity flows over different cavity geometries and Mach numbers. Most previous computations focused on simple rectangular cavities. However, the geometrical configuration of aircraft landing gear and weapon bays includes doors installed at the edge of the cavity, which is more complicated than simple rectangular cavities. Cavities with doors, which involve more complicated flow phenomenon, have not been sufficiently investigated.

The present study investigates cavity flow using the DDES method based on the S-A [18] one-equation model. CFD calculations are performed on the M219 cavity [19], which has a W/D ratio of 1 and an L/D ratio of 5 at Mach 0.85 . Two cavity configurations, clean cavity and cavity with doors, are used. The clean cavity results are evaluated through comparisons of SPL spectra with existing experimental results and the modified Rossiter semi-empirical formula. The results with doors are analyzed by comparing with the clean cavity configuration.

\section{Turbulence Model-DDES}

The one-equation Spalart-Allmaras (S-A) model can be written as

$$
\frac{\partial \widetilde{v}}{\partial \mathrm{t}}+u_{i} \frac{\partial \widetilde{v}}{\partial x_{i}}=\frac{1}{\sigma}\left[\nabla \cdot((v+\tilde{v}) \nabla \tilde{v})+C_{b 2}(\nabla \tilde{v})^{2}\right]+P(\tilde{v})-D(\tilde{v})
$$

where the dissipation is defined as

$$
D(\tilde{v})=C_{w 1} f_{w}\left(\frac{\widetilde{v}}{d}\right)^{2}
$$

and the production term

$$
P(\tilde{v})=C_{b 1} \tilde{v} \widetilde{\Omega} .
$$

$\tilde{v}$ is turbulence variable, $v$ is kinematic viscosity. $\sigma, c_{b 1}, c_{b 2}$ and $\kappa$ are constants. The vorticity variable is given by

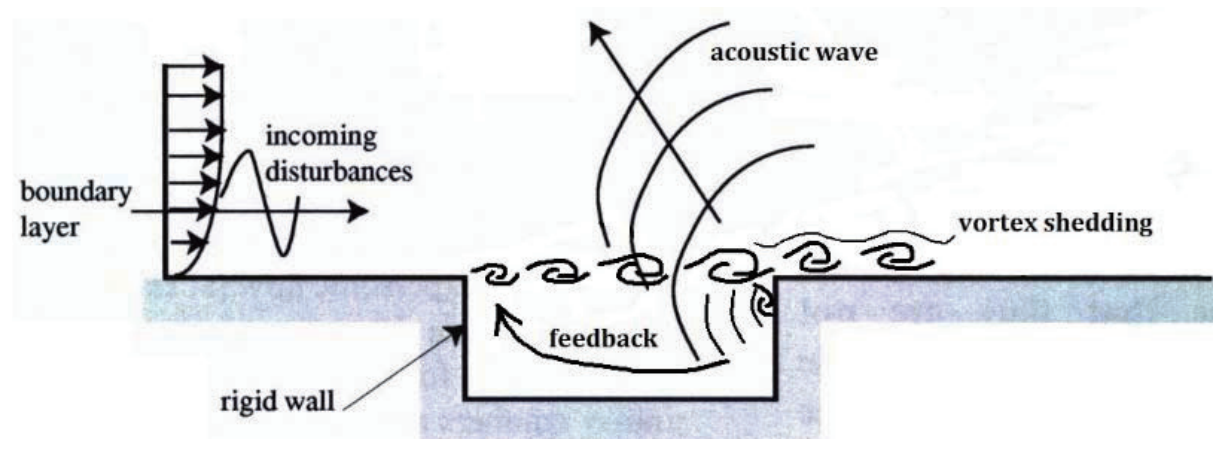

Fig. 1. Schematic of feedback mechanism 


$$
\widetilde{\Omega}=|\Omega|+\frac{\widetilde{v}}{\kappa^{2} d^{2}} f_{v 2}
$$

Functions $f_{w}$ and $f_{v 2}$ are defined to induce turbulence viscosity near the wall. $d$ refers to the distance from wall.

When we convert this standard S-A model into a hybrid RANS/LES form called a Detached Eddy Simulation (DES), $d$ in Eq. (3) will be replaced by

$$
\tilde{d}=\min \left(d, C_{D E S} L_{g}\right)
$$

The empirical constant $C_{D E S}$ has a value of $0.65 . L_{g}$ is a grid length scale defined as

$$
L_{g}=\max (\Delta x, \Delta y, \Delta z)
$$

$\Delta x, \Delta y$ and $\Delta z$ are the local grid lengths. Consequently, the RANS method based on the one-equation S-A model will be adopted in the region near the wall, including the whole boundary layer as $\tilde{d}=d$. When the region is far from the wall, $\tilde{d}=C_{D E S} L_{g}$, and the dissipation is determined by the local grid length. Once the dissipation and production terms reach a balance, it is found from Eq. (2) that $\tilde{v}$ is in direct proportion to $\widetilde{\Omega} d^{2}$, that is,

$$
\tilde{v} \propto \widetilde{\Omega} L_{g}{ }^{2}
$$

Eq. (8) has the same characteristics as the model defined by Smagorinsky [20] as a sub-grid scale model used in the LES method. For a typical RANS grid with a high aspect ratio in the boundary layer, the wall-parallel grid spacing usually exceeds the boundary layer thickness, so Eq. (7) will ensure that the DES model is in RANS mode for the entire boundary layer. However, in the event of a dense grid in all directions, the DES limiter can activate the LES mode inside the boundary layer, where the grid is not fine enough to sustain the LES requirement. Therefore, a new formulation called the Delayed Detached Eddy Simulation (DDES) is presented to preserve the RANS mode throughout the boundary layer.

The length scale $\tilde{\mathrm{d}}$ in DDES is re-defined as

$$
\widetilde{d}=d-f_{d} \max \left(0, d-C_{D E S} L_{g}\right)
$$

where $f_{d}$ is given by $f_{d}=1-\tanh \left(\left[8 r_{d}\right]^{3}\right)$

$r_{d}$ can be considered as a ratio of the turbulence length scale and the wall distance. $f_{d}$ is designed to be 1 in the LES region where $r_{d} \ll 1$, and $f_{d}=0$ elsewhere. As a result, the transport of flows from a region with a large value of eddy viscosities into a region of relatively small strain could cause the DDES model to switch the mode earlier than DES, which results in a transition from LES to RANS mode away from the body.

\section{Simulation Model}

Two test cases were employed in the present numerical analysis. The first model is a clean cavity while the second has doors attached on both sides of the cavity. The cavity, which is the same as the experimental case used by QinetiQ [21], has dimensions of length $\mathrm{L}=508 \mathrm{~mm}$, and depth and width $\mathrm{D}=\mathrm{W}=101.6 \mathrm{~mm}$. The whole testing area is a flat plate with length $1828.8 \mathrm{~mm}$ and width $304.8 \mathrm{~mm}$. The cavity is located $787.4 \mathrm{~mm}$ downstream of the plate with the center line offset $25.4 \mathrm{~mm}$ from the plate center line. The bay doors in the second case are simplified to be a rectangular plate, with height half the cavity width, length equal to the cavity, and thickness $9.525 \mathrm{~mm}$. The dimension of the two simulation models are shown in Fig. 2.

Ten equally spaced Kulite pressure sensors on the ceiling of the cavity are used to catch the pressure histories as shown in Fig. 2. The simulations are performed with free stream conditions of $\mathrm{M}_{\infty}=0.85, \mathrm{P}_{\infty}=62940 \mathrm{~Pa}, \mathrm{~T}_{\infty}=270.25 \mathrm{~K}$ and the Reynolds number, based on the cavity length, is 6.785 million.

The computational domain is bounded by the plane of the flat plate and extends 10D to the upper boundary, including the buffer layer, to remove reflections from the boundary. The 3-D computational mesh of each case consists of 3.5 million hexahedral elements including 0.84 million cells $(200 \times 60 \times 70)$ within the cavity. In order to resolve the small vortices in the viscous boundary layer, the grid is generated meticulously on the plate surface and the shear layer over the cavity. The grid is dense enough to typically result in $y+<$

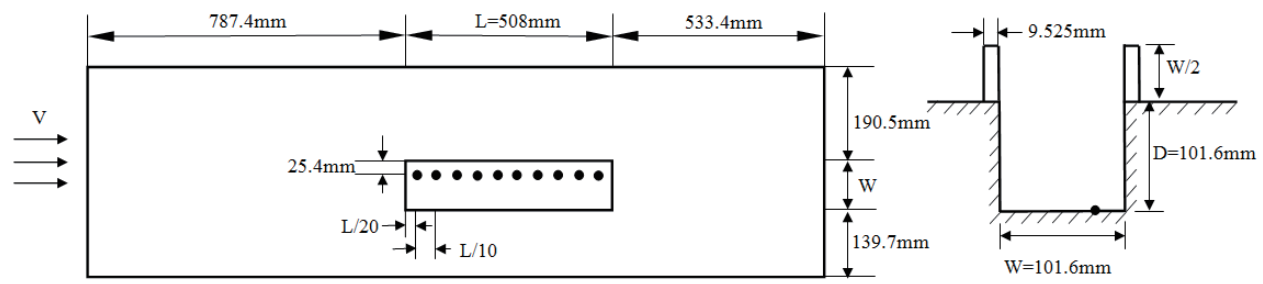

Fig. 2. Clean Cavity and Cavity with Doors 
2 , which is sufficient to resolve the viscosity in the shear layer and near-wall regions. The entire computational domain as well as the mesh for the clean cavity and doors-on cavity are shown in Fig. 3.

Three types of boundary conditions are used in the computation. Because the computational domain is the same as the test rig in the experiment, the inflow comprises of free-stream flow from the wind tunnel. As a result, the pressure far field conditions with $\mathrm{M}=0.85$, pressure $\mathrm{P}=62940 \mathrm{~Pa}$, temperature $\mathrm{T}=270.25 \mathrm{~K}$ and eddy viscosity ratio $\mu_{\mathrm{t}} / \mu_{0}=10$, which are the same as Ashworth's [10], are imposed on the inflow, outflow and upper boundaries. Adiabatic and no-slip wall conditions are applied on the lower surface and on all the cavity walls. Front and back boundaries are set as symmetric boundary conditions similar to the work of Mendonca and Allen [5].

A Green-Gauss cell based finite volume scheme was used with second order implicit time integration and thirdorder MUSCL spatial discretization. The simulation was performed on the Iridis3 compute cluster at the University of Southampton, which is one of the largest computational facilities in the UK, using 12 Intel Nehalem compute nodes each with two 4-core processors. The initial conditions for the DDES were obtained from a steady-state RANS computation with the Spalart-Allamaras (S-A) model. A time-step of $10^{-5}$ seconds with a maximum of 30 iterations per time-step was selected for the DDES transient calculation. The simulation was performed for a total of 0.5 seconds passing the cavity 275 times in the present computation for Mach 0.85. The initial data of 0.3 seconds were discarded to eliminate any transients, leaving the remaining 0.2 seconds for pressure measurements. For the clean cavity case, calculations of 0.5 seconds took approximately 31 days, and the second case with doors took approximately 33 days.

\section{Results and Discussion}

The results consist of two parts: the root mean square pressure $\left(\mathrm{P}_{\mathrm{rms}}\right)$ along the cavity ceiling and the sound pressure level (SPL) used as functions of frequency at locations K20 to K29 respectively for both cases. The first case utilizing the clean cavity can be compared with the experimental results from QinetiQ. Since there are no experimental data available for the doors-on cavity case, the comparison will be made with the first case and the modified semi-empirical Rossiter formula.

The root mean square pressure, $\mathrm{P}_{\text {rms }}$, was taken from the cavity ceiling of both simulations and the experiment occurred at ten locations from k20 on the front part of the cavity to k29 on the rear of the cavity as shown in Fig. 4 . The pressure fluctuations for the cavity flow is triggered by the shear layer developing over the cavity that impacted on the rear wall of the cavity, generating lots of vortices and causing pressure feedback while increasing the instability of the shear layer [3]. The pressure field within the cavity is thus strongly coupled with the shear layer over the cavity. As a result, the distribution of $\mathrm{P}_{\mathrm{rms}}$ could be a reflection of the instability of the shear layer over the cavity.

As seen in Fig. 4, the $\mathrm{P}_{\mathrm{rms}}$ for the clean cavity configuration are relatively low on the front part of the cavity and more intense on the rear part in both simulation and experiment. The computational results of the clean cavity have the same trend as the experiment. Deviations between simulation results and experiment are normally within 300Pa except for the position of $\mathrm{x} / \mathrm{L}=0.75$ close to $600 \mathrm{~Pa}$. Both experiment and simulation results show that the minimum of $\mathrm{P}_{\mathrm{rms}}$ appears at the position of $\mathrm{x} / \mathrm{L}=0.15$ instead of at the very front $\mathrm{x} / \mathrm{L}=0.05$. The reason for this is that the flow, after impact on the rear wall, will transfer to the front and will be stopped by the front wall of the cavity, which will raise the $\mathrm{P}_{\text {rms }}$ of the very front region to slightly above normal. The simulated results for the doors-on cavity are much larger than the clean cavity results. This means that the pressure fluctuation is more intense in the doors-on cavity, and also implies that there are larger disturbances in the shear layer compared with the clean cavity. The reason could be that the cavity doors stop the shear layer from flowing to the span-wise direction, resulting in more flows impacting the rear door into the cavity and engaging in the feedback process. As a result, the presence

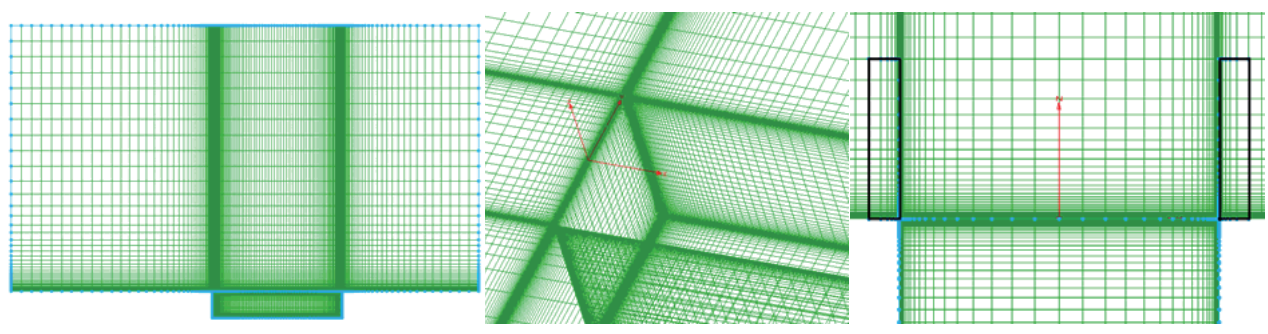

Fig. 3. Mesh for the Whole Computational Domain and Doors 
of side doors will lead to more intense pressure fluctuations inside the cavity.

The sound resonances identified as acoustic tones are caused by pressure fluctuations in the cavity. The time series of $\mathrm{P}_{\mathrm{rms}}$ was analyzed by means of Fast Fourier Transform providing SPL as a function of frequency. SPL is defined by

$$
\mathrm{SPL}=20 \lg \left(\frac{\mathrm{P}_{\mathrm{rms}}}{\mathrm{P}_{\mathrm{ref}}}\right)
$$

where $\mathrm{P}_{\text {ref }}=2 \times 10^{-5} \mathrm{pa}$ is the minimum audible sound pressure variation. Fig. 5 shows the SPLs at four Kulite locations, $\mathrm{x} /$
$\mathrm{L}=0.05,0.35,0.65$ and 0.95 , from the inflow edge to the exit edge for both cases.

The first observation is that the SPLs at the rear of the cavity are much higher than at the front as expected. This is because the high speed flow impacts the back wall of the cavity, making the rear the most unstable region with lots of acoustic waves generated. It is well known that there are four SPL peaks corresponding to four Rossiter frequencies [3], as seen in the experiment. The second and third peaks are outstanding as dominant peaks in the spectrum. The first mode is generally under predicted with a range

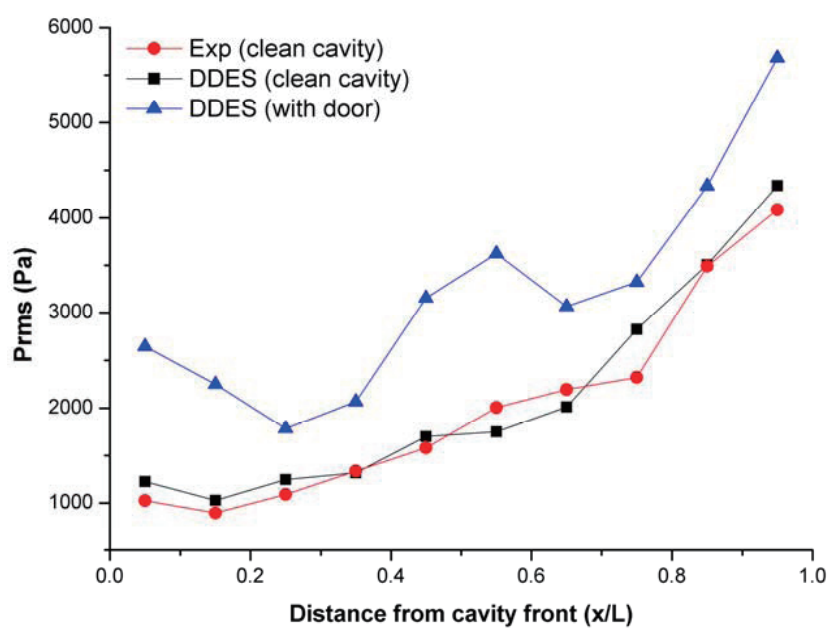

Fig. 4. Prms along the Cavity Ceiling
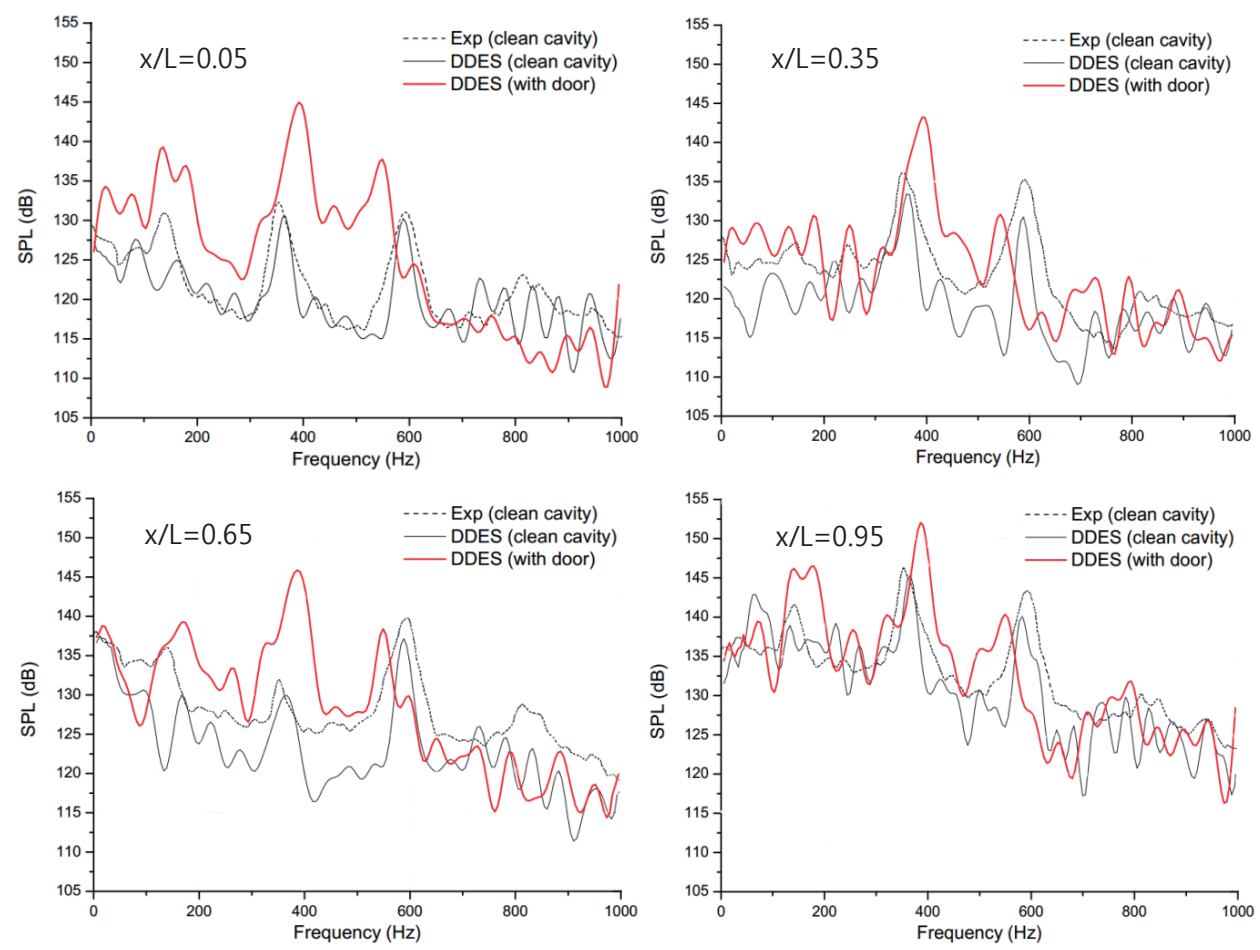

Fig. 5. Sound Pressure Levels at $x / L=0.05,0.35,0.65$ and 0.95 
from 2-6dB. The second mode is well predicted with an under-prediction below $3 \mathrm{~dB}$. The third mode is rather well predicted with an under-prediction deviation within $4 \mathrm{~dB}$. There is peak splitting phenomenon [22] in the fourth mode of the spectrum, which means that it is not easily predicted. The fourth mode is under predicted by no more than $3 \mathrm{~dB}$. The resonant frequencies are very well predicted for the dominant second and third modes within $10 \mathrm{~Hz}$, and for the weaker first and fourth modes within $60 \mathrm{~Hz}$. In general, the DDES computational results match the experimental results well in the first three modes. Therefore, the DDES method performs very well.

Comparisons of DDES for the clean cavity and the cavity with doors reveal that the SPLs inside the cavity as well as the magnitude of tones are increased by the doors at all four locations. Due to the presence of doors, pressure amplitudes are magnified, resulting in a rise of SPLs by as much as $15 \mathrm{~dB}$, especially for the first and second modes from the clean cavity. The amplitude of the first mode increases $5-10 \mathrm{~dB}$, and the corresponding frequency changes within $30 \mathrm{~Hz}$. It should be noted that the amplitude of the first mode increases significantly at the location of $\mathrm{x} / \mathrm{L}=0.05$ and 0.95 where the flow impacted the rear wall, leading to the peak splitting phenomenon. The amplitude of the second mode is magnified the most by as much as $15 \mathrm{~dB}$, with the frequency shifted to around $50 \mathrm{~Hz}$ higher. The amplitude of the third mode remains at the same level except for the position of $x / L=0.05$, where the SPL increases by about $6 \mathrm{~dB}$, and the frequency is shifted by $50 \mathrm{~Hz}$ lower. The computational results of the fourth mode are unable to show the peak as clearly as in the clean cavity case. From the overall trend, it is speculated that the SPLs at high frequency regions change less. In general, the SPL inside the doors-on cavity are higher than in the clean cavity case. It may be that the cavity doors stop the shear layer from flowing in the span-wise direction, resulting in more flows impacting the rear wall into the cavity as well as the feedback process. As a result, the presence of side doors will lead to more intense pressure fluctuations inside the cavity, causing a dramatic increase in SPLs. In order to fully analyze the noise level in the clean cavity and the effect of doors, the SPL results, including four mode frequencies and the corresponding amplitudes at location $\mathrm{x} /$ $\mathrm{L}=0.95$, are quantified in Tab. 1 .

It can be seen from Tab. 1 that the mode frequencies predicted by the modified Rossiter formula match the experiment pretty well, especially on the 3rd and 4th modes. The frequency deviations between the experiment and the DDES on the clean cavity case are no more than $9 \%$ except in the 1st mode. According to Xiaoxian Chen [23], inadequate computing time may be the reason for the unsatisfactory mode frequency prediction especially for the 1st mode, as accurate prediction would require long integration times. Because the first mode is strongly involved in the instability of the shear layer, the RANS computation region could also influence the precision of the first mode. Generally, the tone amplitudes of all four modes are predicted exactly, with the deviations no more than $2.4 \mathrm{~dB}$. After two doors are installed on the side edge, the frequency of the 2nd mode is shifted up to higher values while the effect on the 3rd mode is the opposite. The amplitude of the dominant 2nd mode increases by over $5 \mathrm{~dB}$ in comparison with the clean cavity case in both the DDES and the experiment. The amplitude of the 1st mode is increased by $4 \mathrm{~dB}$, which is less than the $2 \mathrm{nd}$ mode. The amplitudes of the 3rd and 4th modes basically remain on the same level, with small change within $2 \mathrm{~dB}$. The DDES simulation indicates that doors can force the vortices into the cavity, enlarging the impingement with the rear wall and enhancing the oscillation of the shear layer over the cavity. This results in an increase in SPLs especially for the 2nd dominant mode.

In order to observe the $3 \mathrm{D}$ unsteady flow field, the computed velocity flow field contours of velocity magnitude are illustrated in Fig. 6, and the vorticity magnitude contours are shown in Fig. 7. The shear layer coming off the cavity

Table 1. Mode Frequency and Amplitude Comparisons at $\mathrm{x} / \mathrm{L}=0.95$

\begin{tabular}{clcccc}
\hline & & Mode & 2nd & 3rd & 4th \\
\hline Modified Rossiter & Frequency [Hz] & 162 & 378 & 595 & 812 \\
Experiment & Frequency [Hz] & 142 & 353 & 592 & 813 \\
(Clean Cavity) & Amplitude [dB] & 141.57 & 146.27 & 143.37 & 130.17 \\
DDES & Frequency [Hz] & 68 & 367 & 589 & 784 \\
(Clean Cavity) & Amplitude [dB] & 142.95 & 145.52 & 141.04 & 130.03 \\
DDES & Frequency [Hz] & 141 & 386 & 551 & 791 \\
(Cavity with Doors) & Amplitude [dB] & 146.34 & 152.11 & 140.46 & 131.91 \\
\hline
\end{tabular}


leading edge oscillates at different cross sections. It can be seen from the velocity magnitude contours that the fluctuations of the shear layer at the rear section are more intense than at the front sections in both cases. In the clean cavity case, the shear layer not only spans the mouth of the cavity but also spreads to the span-wise direction, resulting in oscillations around side edges. In contrast, the doors in the second case force the incoming flow into the cavity, which results in more powerful impact on the rear wall. Comparing with the clean cavity case as seen in Fig. 7, the interaction between the shear layer and the rear wall gives rise to the instability of the shear layer. The shear layer instability is coupled with the pressure waves generated in the cavity. As a result, the acoustic tones at discrete frequencies analyzed in the above section are amplified in the doors-on case.

\section{Conclusions}

DDES computations have been conducted for an opencavity flow using the S-A one equation model. The open cavity immersed in a free stream at a Mach number of 0.85

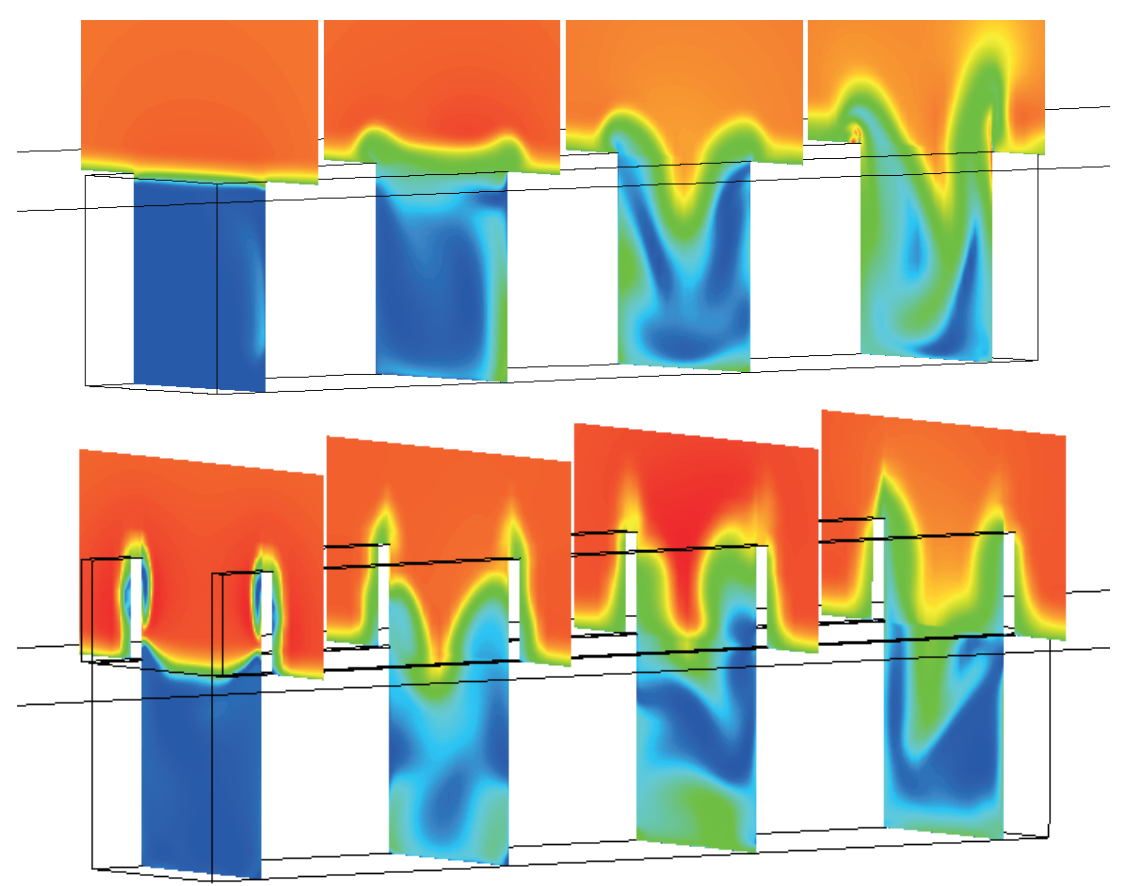

Fig. 6. Velocity Magnitude Contours for Clean Cavity (up) and with Doors (down)
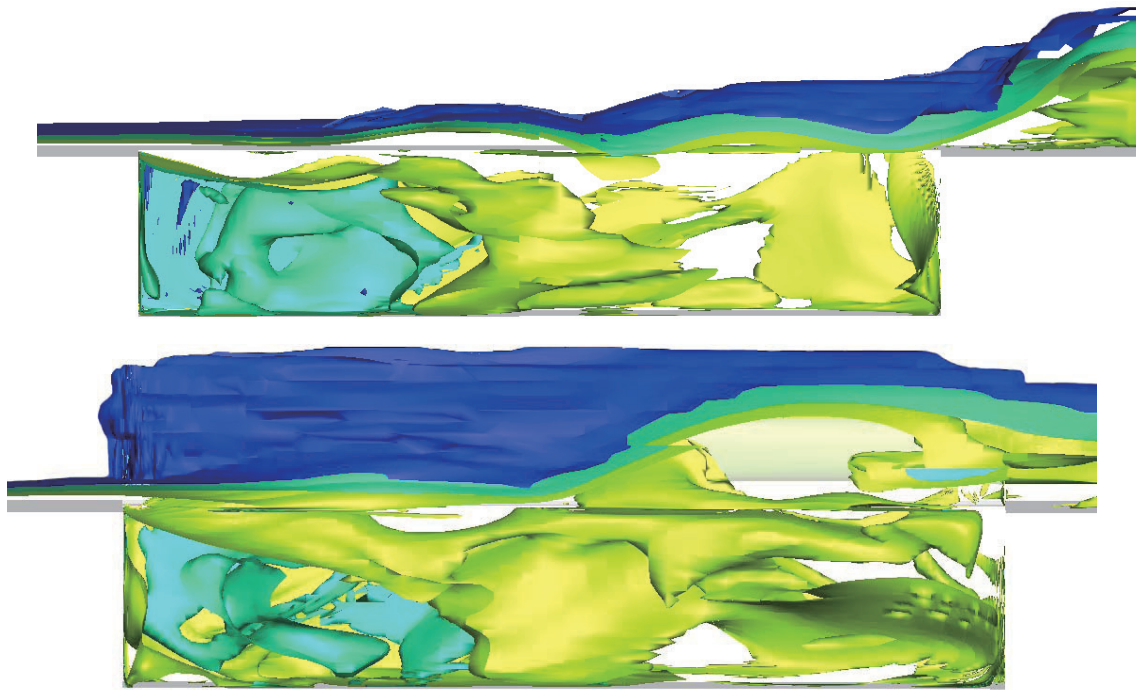

Fig. 7. Vorticity Magnitude Contours for Clean Cavity (up) and with Doors (down) 
has an aspect ratio of 5:1:1. Two configurations, clean cavity and cavity with doors installed on both sides, are simulated. Both simulations show that the SPLs at the rear of the cavity are much higher than at the front. This is due to the shear layer moving up and down with breakup and impingement on the rear of the cavity. The interaction between the shear layer and the rear wall gives rise to two consequences. First, a recirculation flow is formed in the cavity due to the entrainment of the shear layer and the flow reverses after the impact on the rear wall. Second, the interaction provides an effective feedback that amplifies the shear layer instability. The DDES computational results show the same trend as the experimental results for the amplitude of all tones, but the frequency of the 1st mode is under predicted. Comparisons of DDES for the clean cavity and the doors-on cavity reveal that the SPLs inside the cavity as well as the magnitude of tones are amplified by the side doors. Cavity doors force the entire shear layer containing all scales of vortices into the cavity, enlarging the impingement with the rear wall and enhancing the oscillation of the shear layer over the cavity. This results in an increase in SPLs especially for the 2nd dominant mode.

\section{Acknowledgement}

This paper is part of a project funded by the Priority Academic Program Development of Jiangsu Higher Education Institutions (PAPD).

This work is also supported by the Funding of Jiangsu Innovation Program for Graduate Education KYLX_0296, and the Fundamental Research Funds for the Central Universities.

\section{References}

[1] K. Karamcheti, Acoustic Radiation from TwoDimensional Rectangular Cutouts in Aerodynamic Surfaces, Technical Report 3487, California Institute of Technology, August 1955.

[2] Mark H. Morton, Craig D. Hampson, and Ric A. Alexander, "Final vibration and Acoustic Loads Development for Certification of the F-22 Advanced Tactical Fighter", Proceeding of the 49th AIAA/ASME/ASCE/AHS/ASC Structures, Structural Dynamics and Materials Conference, Schaumburg, IL, USA, 2008.

[3] Rossiter, J.E., Wind Tunnel Experiments on the Flow over Rectangular Cavities at Subsonic and Transonic Speeds, Reports and Memoranda 3438, Aeronautical Research Council, London, 1964.6.
[4] Heller. H., Holmes. D, and Covert. E, "Flow Induced Pressure Oscillations in Shallow Cavities", Journal of Sound and Vibration, Vol. 18, No. 4, 1971, pp. 545-553.

DOI: 10.1016/0022-460X(71)90105-2

[5] Mendonca, F., Allen. R., de Charentenay. J, and Kirkham. D, "CFD Prediction of Narrowband and Broadband Cavity Acoustics at $\mathrm{M}=0.85$ ", Proceeding of the 9th AIAA/ CEAS Aeroacoustics Conference and Exhibit, Hilton Head, South Carolina, USA, 2003.

[6] Shia Hui Peng, "Simulation of Turbulent Flow Past a Rectangular Open Cavity Using DES and Unsteady RANS", Proceeding of the 24th Applied Aerodynamics Conference, San Francisco, California, USA, 2006.

[7] Arina. R. and Ceresola. N., "Numerical Simulation of the Aerodynamic Flow Field and Radiated Noise of a Transonic cavity", Proceeding of the European Conference on Computational Fluid Dynamics, ECCOMAS CFD, 2006.

[8] Li. Z., Debashis Basu. and Hamed. A., "Numerical Simulation of Sidewall Effects on the Acoustic Field in Transonic Cavity", Proceeding of the 45th AIAA Aerospace Sciences Meeting and Exhibit, Reno, Nevada, Jan 2007.

[9] Larcheveque. L., Sagaut. P., Le. T., "Large-Eddy Simulations of Flows in Weapon Bays", Proceeding of the 41st Aerospace Sciences Meeting and Exhibit, Reno, Nevada, Jan 2003.

[10] Ashworth. R., "Prediction of acoustic resonance phenomena for cavities using detached eddy simulation". Proceeding of the Royal Aero Society Conference, QinetiQ, UK, 2004.

[11] Allen. R. and Mendonca. F., "DES Validation of Cavity Acoustics over the Subsonic to Supersonic Range", Proceeding of the 10th AIAA/CEAS Aeroacoustics Conference, Manchester, United kingdom, 2004.

[12] Boydston. J., Squires. K. and Forsythe. J., "Detached Eddy Simulation of High Reynolds Number Flow over a Rectangular Cavity", Proceeding of the 46th AIAA Aerospace Sciences Meeting and Exhibit, Reno, Nevada, Jan 2008.

[13] Travin. A., Shur. M., Strelets. M. and Spalart. P., "Physical and Numerical Upgrades in the Detached Eddy Simulation of Complex Turbulent Flows", Advances in LES of Complex Flows, edited by P. Friedrich and W.Rodi, Kluwer Academic Publishers, 2002, pp. 239-254.

[14] Spalart. P. R., Jou. W. H., Strelets. M. and Allmaras. S. R., "Comments on the Feasibility of LES for Wings, and on a Hybrid RANS/LES Approach," Advance in DNS/LES, Proceeding of the 1st AFOSR International Conference on DNS/LES, Greyden Press, Columbus, OH, 1997.

[15] Squires. K. D., "Detached Eddy Simulation: Current Status and Perspectives," Proceedings of Direct and LargeEddy Simulation-5, Kluwer Academic, Norwell, MA, 2004. 
[16] Zhang, J., Morishitaf. E., Okunukil. T. and Itoh. H., "Experimental and Computational Investigation of Supersonic Cavity Flows", Proceeding of the 10th AIAA/ NALNASDA-ISAS International Space Planes and Hypersonic Systems and Technologies Conference, Kyoto, Japan, 2001.

[17] Chung. K., "Characteristics of Compressible Rectangular Cavity Flows", Journal of Aircraft, Vol. 40, No. 1, 2003, pp. 137-142.

DOI:10.2514/2.3068

[18] Spalart. P. R. and Allmaras. S. R., "A One-Equation Turbulence Model for Aerodynamic Flows," Proceeding of the 30th Aerospace Sciences Meeting and Exhibition, Reno, NV, USA, Jan 1992.

[19] Henshaw. M.J. de C., "M219 cavity case-Verification and Validation Data for Computational Unsteady Aerodynamics", Tech. Rep. RTO-TR-26, AC/323 (AVT) TP/19,
QinetiQ, UK, 2000.

[20] Smagorinsky. J., "General Circulation Experiments with the Primitive Equations", Monthly Weather Review, Vol. 91, No. 3, 1963, pp. 99-164.

[21] G.W. Foster, J.A. Ross, and R.M. Ashworth, "Weapon Bay Aerodynamics Wind Tunnel Trials and CFD Modeling by QinetiQ UK", Proceeding of the RTO/AVT Symposium on Flow-Induced Unsteady Loads and the Impact on Military Applications, Budapest, Hungary, 2005.

[22] Rowley. C. W. and Williams. D. R., "Dynamics and Control of High Reynolds number Flow over Open Cavities", Annual Review of Fluid Mechanics, Vol. 38, 2006, pp. 251-276.

DOI: 10.1146/annurev.fluid.38.050304.092057

[23] Xiaoxian Chen, Neil D. and Sandham, Xin Zhang, "Cavity Flow Noise Predictions", Tech. Rep. MSTARR-DARP, AFM-07/05, Southampton, UK, 2007. 